\title{
Prävention der beatmungsassoziierten Pneumonie: höchste Zeit zu handeln!
}

\author{
Hans Georg Bone
}

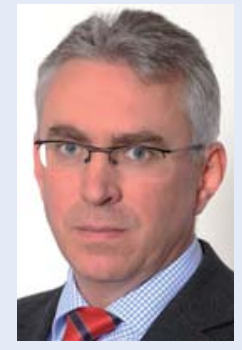

Hans Georg Bone
D

ie Bundesbürger wurden Anfang 2014 aufgeschreckt: Die Presse berichtete umfangreich über die Ergebnisse des AOK-Krankenhausreports 2014 [1]. In diesem Krankenhausreport wurde von 19000 vermeidbaren Todesfällen durch Fehler in deutschen Krankenhäusern ausgegangen. Ein wesentlicher Aspekt bei der Diskussion um die von der AOK vorgestellten Daten waren die nosokomialen Infektionen, die nach Angaben des Berichts ca. $4 \%$ aller stationären Patienten betreffen. Nach Ansicht der Presse könnten diese nosokomialen Infektionen größtenteils durch einfache Präventionsmaßnahmen verhindert werden. In einem Teil der Presse wurden auch noch wesentlich ungünstigere Daten diskutiert, nach denen sich im Jahr in Deutschland 1 Mio. Patienten in Krankenhäusern aufgrund von mangelnder Hygiene infizieren und 40000 Patienten an diesen Infektionen pro Jahr in Deutschland versterben würden [2]. Der Patientenbeauftragte der Bundesregierung, Herr Staatssekretär Karl-Josef Laumann, sagte daraufhin in einem Interview mit der Westdeutschen Allgemeinen Zeitung am 4.3.2014: „.... Hygiene im Krankenhaus ist Patientenschutz, und zwar einer der wichtigsten Eckpfeiler, die es im Patientenschutz überhaupt gibt. Man wird in einem Krankenhaus Keime nie ausschließen können, aber man muss alles tun, dass der Verbreitung von Keimen dort Einhalt geboten wird...." Im Lichte dieser öffentlichen Diskussionen erhält der Artikel „Prävention der beatmungsassoziierten Pneumonie" von Lewalter, Lemmen und Scheithauer in diesem Heft eine besondere Bedeutung. Die nosokomiale Pneumonie und insbesondere die beatmungsassoziierte Pneumonie (ventilator-associated pneumonia: VAP bzw. tubusassoziierte Pneumonie) ist unverändert eine bedrohliche Komplikation im Verlauf einer Krankenhausbehandlung. Nosokomiale untere Atemwegsinfektionen liegen auf Platz 3 der nosokomialen Infektionen in Deutschland, nach den Harnwegsinfektionen und den postoperativen Wundinfektionen [3]. In Deutschland geht man bei den beatmungsassoziierten Pneumonien von einer Häufigkeit von 5,4 Fällen pro 1000 Tagen invasiver Beatmung aus [4]. Bei nichtinvasiver Beatmung ist die Häufigkeit mit 1,2 Fällen pro
1000 Tagen Beatmung deutlich niedriger [5]. Das Auftreten von beatmungsassoziierten Pneumonien ist verbunden mit

- einem längeren Intensivaufenthalt,

- einer höheren Morbidität und Mortalität,

- einem höheren Ressourcenverbrauch,

- höheren Behandlungskosten [6,7].

Also besteht nicht nur aufgrund der vermehrten Presseberichterstattung, sondern auch aus medizinischen, humanitären und ökonomischen Gründen dringender Handlungsbedarf zur Vermeidung beatmungsassoziierter Pneumonien. Eine große Gruppe unterschiedlicher deutscher Fachgesellschaften, u. a. die

- Deutsche Gesellschaft für Anästhesiologie und Intensivmedizin,

- Deutsche Gesellschaft für Infektiologie,

- Deutsche Gesellschaft für Hygiene und Mikrobiologie,

- Deutsche Gesellschaft für Pneumologie und

Beatmungsmedizin,

- und die Paul-Ehrlich Gesellschaft,

hat gemeinsam den Kraftakt auf sich genommen und eine S-3-Leitlinie zur Epidemiologie, Diagnostik und Therapie der nosokomialen Pneumonie beim Erwachsenen erstellt, die vor kurzem publiziert wurde [8]. Im Text der Leitlinie heißt es: „Zur Prävention nosokomialer Pneumonien, ... verweisen wir auf die entsprechenden Leitlinien bzw. Empfehlungen des Robert Koch-Instituts, der Deutschen Gesellschaft für Pneumologie und Beatmungsmedizin und der Deutschen Sepsisgesellschaft.“ Die Empfehlungen der Kommission für Krankenhaushygiene und Infektionsprävention (KRINKO) zur Prävention der nosokomialen Pneumonie sind dann aber erst ein Jahr später publiziert worden [5]. Die zentrale Botschaft sowohl der KRINKO-Empfehlungen wie auch der Empfehlungen aus der Übersichtsarbeit von Lewalter et al. in diesem Heft ist die gleiche: Für eine erfolgreiche Prävention von beatmungsassoziierten Pneumonien ist ein ganzes Maßnahmenbündel erforderlich und die Umsetzung dieser Maßnahmen muss immer wieder kontrolliert werden. Die einzelnen Bestandteile des Maßnahmenbündels sind häufig nicht aufwendig in der Umsetzung, wie z.B. 
eine verbesserte Händehygiene, eine regelmäßige Cuff- Literatur

druck-Kontrolle, eine frühe enterale Ernährung oder eine hygienische Mundpflege. Wichtig ist jedoch nicht nur darüber zu reden, sondern zügig zu handeln und diese Maßnahmenbündel umzusetzen!

\section{Korrespondenzadresse}

Prof. Dr. med. Hans-Georg Bone

Chefarzt des Zentrums für Anästhesiologie, Intensivmedizin und Schmerztherapie

Klinikum Vest GmbH

Dorstener Straße 151

45657 Recklinghausen

E-Mail: Hans-Georg.Bone@Klinikum-Vest.de

1 http://www.aok-bv.de/presse/pressemitteilungen/2014/ index_11342.html

2 Brand K. Zahl der Infektionstoten doppelt so hoch wie zugegeben. In: Westdeutsche Allgemeine Zeitung; 26.02.2014

3 Robert-Koch-Institut. Deutsche Daten im Rahmen der ersten europäischen Prävalenzerhebeung zum Vorkommen nosokomialer Infektionen und zur Antibiotikaanwendung. Epid Bull 2012; 26: 239-240

4 Kohlenberg ASF, Behnke M, Gastmeier P. Pneumonia associated with invasive and noninvasive ventilation: an analysis of the German nosokomial infection surveillance system database. Intensive Care Med 2010; 36: 971-978

5 Kommission für Krankenhaushygiene und Infektionsprävention (KRINKO) am Robert-Koch-Institut. Prävention der nosokomialen beatmungsassoziierten Pneumonie. Bundesgesundheitsblatt Gesundheitsforschung Gesundheitsschutz 2013; 56: $1578-1590$

6 Safdar NDC, Collard HR, Saint S. Clinical and economic consequences of ventilator-associated pneumonia: a systematic review. Crit Care Med 2005; 33: 2184-2193

7 Hugonnet SEP, Borst F, Maricot P et al. Impact of ventilatorassociated pnemonia on resource utilization and patient outcome. Infect Control Hosp Epidemiol 2004; 25: 1090 - 1096

8 Dalhoff K, Abele-Horn M, Andreas S et al. Epidemiologie, Diagnostik und Therapie erwachsener Patienten mit nosokomialer Pneumonie. S-3 Leitlinie der Deutschen Gesellschaft fur Anasthesiologie und Intensivmedizin e.V., der Deutschen Gesellschaft fur Infektiologie e.V., der Deutschen Gesellschaft fur Hygiene und Mikrobiologie e.V., der Deutschen Gesellschaft fur Pneumologie und Beatmungsmedizin e.V. und der Paul-Ehrlich-Gesellschaft fur Chemotherapie e.V. Pneumologie 2012; 66: $707-765$ 